\title{
HD 152248: Evidence for a colliding wind interaction*
}

\author{
H. Sana, G. Rauw ${ }^{\star \star, \dagger}$, and E. Gosset ${ }^{\star \star \star, \dagger}$ \\ Institut d'Astrophysique et de Géophysique, Université de Liège, 5 avenue de Cointe, 4000 Liège, Belgium
}

Received 18 December 2000 / Accepted 7 February 2001

\begin{abstract}
We present the results of a four year spectroscopic campaign on the early-type binary system HD 152248 . Our analysis yields a new classification as O7.5III(f) + O7III(f), in contradiction with previous classifications of the components as supergiants. We derive improved orbital elements and the corresponding masses $\left(M_{1}=29.6 M_{\odot}\right.$ and $M_{2}=29.9 M_{\odot}$ ) are somewhat larger than previously reported in the literature, although they are still significantly lower than the ones expected from evolutionary tracks. Both components of the system are close to filling their Roche lobe at periastron passage. We also investigate the equivalent width variations of the lines of the two components and we discuss their relation to the Struve-Sahade effect. Finally, we show that the line profile variability of the He II $\lambda 4686$ and $\mathrm{H} \alpha$ lines is consistent with a strong wind interaction between the two stars, quite as predicted by current colliding wind models.
\end{abstract}

Key words. binaries: close - binaries: spectroscopic - stars: early-type - stars: fundamental parameters - stars: individual: HD 152248 - stars: winds, outflows

\section{Introduction}

Early-type stars of spectral-type O or Wolf-Rayet (WR) undergo a considerable mass loss through their powerful winds. Within a binary system, we expect that the winds from both stars collide, forming a wind interaction region that can manifest as enhanced X-ray emission. This X-ray emission from the shock can display a phase-locked modulation due to the variation of the line-of-sight opacity or due to a changing separation between the stars in eccentric binaries (Pittard \& Stevens 1997). The wind-wind collision can also contribute to the formation of optical emission lines through the recombination process (e.g. Thaller 1997).

Over the last twenty years, the interest in wind interaction has grown, and numerous studies both from theoretical (e.g. Stevens et al. 1992; Walder 1998; Pittard 2000) and observational (e.g. Corcoran 1996; Rauw et al. 1999; Hill et al. 2000) points of view have been

\footnotetext{
Send offprint requests to: H. Sana,

e-mail: sana@astro.ulg.ac.be

* Based on data collected at the European Southern Observatory (La Silla, Chile) and at the Cerro Tololo InterAmerican Observatory (Cerro Tololo, Chile).

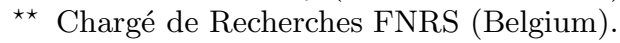

$\star \star \star$ Chercheur Qualifié FNRS (Belgium).

$\dagger$ Visiting Astronomer, CTIO, National Optical Astronomy Observatories (NOAO). NOAO is operated by the Association of Universities for Research in Astronomy, Inc. under contract with the National Science Foundation.
}

undertaken. These studies demonstrate that considerable insight into the mass loss process can be gained from a detailed investigation of the wind interaction phenomenon. In addition, binary systems provide precious astrophysical laboratories allowing a direct determination of the fundamental parameters of the stars that are crucial in order to test evolutionary models and to probe the impact of binarity on the evolution of the components of the system.

HD 152248 is an $\mathrm{O}+\mathrm{O}$ binary system that belongs to the young open cluster NGC 6231 which is considered to be the nucleus of the rich ScO OB1 association. Situated at a distance of $1.99 \mathrm{kpc}$ (Baume et al. 1999), this cluster contains a number of interesting single $\mathrm{O}$ stars as well as $\mathrm{O}$ binaries and a WR+O system (WR79). Not only is the HD 152248 system one of the brightest objects of the cluster $(V=6.06$, Baume et al. 1999$)$ but it also appears from ROSAT X-ray observations to be the brightest X-ray source in NGC 6231. Corcoran (1996) has further shown that the ROSAT X-ray flux from HD 152248 displays phase-locked variations, though the poor phase coverage of the ROSAT data did not allow elucidation of their origin. On these grounds, NGC 6231 has been selected as a target for the XMM X-ray observatory as part of the Liège project for guaranteed time. The pointings will be centered on HD 152248 for a total duration of $180 \mathrm{ksec}$, consisting of six $30 \mathrm{ksec}$ exposures in order to adequately sample the orbital variations.

In this context, we have undertaken a spectroscopic campaign to monitor the optical counterpart of most of 
the ROSAT X-ray sources of the NGC 6231 cluster. Some preliminary results have already been presented by Sana et al. (2001). The present paper is the first of a series devoted to the O stars in NGC 6231. We present here a complete and detailed spectroscopic study of the HD 152248 system (the main target of our campaign) in the optical domain. One aim is to derive accurate, contemporaneous ephemerides required to correctly interpret the forthcoming XMM data and to clarify the evolutionary status of both components of the system. Another objective of this study is the search for the optical signature of a wind interaction which is suspected to exist within the system because of its high and variable X-ray luminosity.

This paper is organised as follows. The next section is devoted to a review of the main results already published about this object in the literature. In the third section, we describe our spectroscopic observing campaign, the data we collected and the data reduction techniques we applied. The determination of the orbital parameters is presented in the fourth section. The fifth one is devoted to the study of the variations of the equivalent widths of the absorption lines and their link to the Struve-Sahade effect (S-S effect). We next turn to the emission lines displayed by the spectrum of this system. Particular attention is paid to the He II $\lambda 4686$ and $\mathrm{H} \alpha$ lines and to their phase-locked variability. Section 7 is devoted to the discussion of the evolutionary status of the system, and, among other things, we re-investigate its spectral and luminosity classification. Finally, we present a wind-wind interaction model that is consistent with most of the observational features of the emission lines. The last section is devoted to a brief summary of our main results.

\section{Overview}

HD 152248 is a double line $\mathrm{O}+\mathrm{O}$ spectroscopic binary with a period close to six days. It was first reported to be a binary by Struve (1944), although he suggested an orbital period of 3 days. Walborn (1972) quoted a spectral type of $\mathrm{O} 7.5 \mathrm{I}(\mathrm{n})(\mathrm{f}) \mathrm{p}$ for the composite spectrum. Hill et al. (1974, HCB hereafter) managed to separate the primary and secondary components and derived a period of 5.97 days, assuming a zero eccentricity. Levato \& Morrell (1983) confirmed the 6 day period and derived a non-zero eccentricity $(e=0.18 \pm 0.02)$ for the system. A polarimetric study by Luna (1988) constrained the inclination of the orbit between $60^{\circ}$ and $76^{\circ}$, with a preferred value of $71^{\circ}$. The study of the light curve of HD 152248 by Mayer et al. (1992) revealed that the system displays two eclipses of unequal depth and that both minima are not separated by a 0.5 cycle, suggesting a non-zero eccentricity. Based on IUE observations and using a cross-correlation technique, Stickland et al. (1996, ST96 hereafter) derived a period of 5.816083 days, an eccentricity of 0.124 and a mass ratio of 0.941 . The deepest eclipse corresponds to the occultation of the less-massive component. In order to avoid confusion, we choose to adopt the convention of ST96 for the primary and secondary. In the following, we will thus refer to the component occulted during the deepest (i.e. primary) eclipse as the primary star of the system.

Based on the same set of IUE data and using a tomographic reconstruction algorithm to separate primary and secondary spectra, Penny et al. (1999, PGB hereafter) derived a spectral type of O7I+O7I. They also re-analysed the light curve of the system, complementing the Mayer et al. (1992) data set with Hipparcos observations. PGB showed that the system displays ellipsoidal light variations due to tidal deformations. They derived an inclination of $72^{\circ} \pm 3^{\circ}$ for the orbit of the system and polar radii of $R_{1}=13.4 \pm 2.0 R_{\odot}$ and $R_{2}=12.9 \pm 2.0 R_{\odot}$ for the primary and secondary respectively. Combining spectroscopic and photometric results, they computed masses of $M_{1}=24.2 \pm 2.0 M_{\odot}$ and $M_{2}=25.8 \pm 2.0 M_{\odot}$. Howarth et al. (1997) reported that HD 152248 is displaying the Struve-Sahade effect, i.e. that the secondary lines seem to be stronger when the secondary is approaching. However, this result was not confirmed by PGB, who found no evidence for this effect in the strength of the cross-correlation function of the IUE spectra of HD 152248 with their standard star, HD 34078.

\section{Observations and data reduction}

\subsection{Observations}

We have been monitoring the HD 152248 binary for four years (from May 1996 until May 2000), collecting a number of spectra with various instruments at the European Southern Observatory (ESO) and at the Cerro Tololo Inter-American Observatory (CTIO). In this section, we present the general features of the instrumental configuration and data reduction. The campaign we have undertaken represents a total of 69 useful spectra obtained during 49 nights spread over nine separate runs. The main journal of the observations is given in Table 1.

During two runs in May 1996 and March 1997, a set of medium resolution spectra was obtained with the Boller \& Chivens spectrograph (B\&C) fed by the ESO $1.5 \mathrm{~m}$ telescope. The detector was a $2 \mathrm{k} \times 2 \mathrm{k}$ UV flooded Loral-Lesser CCD. The pixel size is $15 \times 15 \mu \mathrm{m}$. We used the holographic grating (ESO grating \#32) with 2400 lines $/ \mathrm{mm}$, providing a spectral resolution of $1.2 \AA$ as measured from the $F W H M$ of the lines of the HeAr comparison spectrum. The covered spectral range extends from $\lambda 3790$ to $\lambda 4765 \AA$.

High resolution spectra of the He I $\lambda 4471, \mathrm{~N}$ III $\lambda \lambda 4634$ 4641 and He II $\lambda 4686$ lines were obtained with ESO's $1.4 \mathrm{~m}$ Coudé Auxiliary Telescope (CAT) at La Silla, using the Coudé Echelle Spectrometer (CES) equipped with the Long Camera (LC, before March 1998) or the Very Long Camera (VLC, after March 1998). The detector used was ESO CCD\#38, a Loral $2688 \times 512$ pixel CCD with a pixel size of $15 \times 15 \mu \mathrm{m}$. The slit width was chosen to achieve a nominal resolving power of $70000-80000$. The effective resolving power as derived from the $F W H M$ of the lines of the ThAr calibration exposures is 65000-75000. Typical 
Table 1. Main journal of the spectroscopic observations of the HD 152248 system. The first column lists the heliocentric Julian date (HJD). The second gives the observational phase as computed from our He I $\lambda 4471 R V$ orbital solution (cf. Table 3 ). Columns 3 and 4 (resp. 5 and 6) provide the He I $\lambda 4471$ line $R V$ (resp. the mean $R V$ ) for the primary and secondary stars, in the "zero systemic velocity" reference frame (cf. text). The number of lines used to compute the mean $R V$ is given in Col. 7 and the last column provides information about the instrumentation used

\begin{tabular}{|c|c|c|c|c|c|c|c|}
\hline \multirow{2}{*}{$\begin{array}{c}\text { HJD } \\
-2450000\end{array}$} & \multirow[t]{2}{*}{$\phi$} & \multicolumn{2}{|c|}{ He I $\lambda 4471-\overline{\gamma_{4471}}$} & \multicolumn{2}{|c|}{$\overline{R V_{\lambda}-\overline{\gamma_{\lambda}}}$} & \multirow[t]{2}{*}{$n$} & \multirow[t]{2}{*}{ Instrument. } \\
\hline & & $R V_{1}$ & $R V_{2}$ & $R V_{1}$ & $R V_{2}$ & & \\
\hline 205.859 & 0.851 & 207.4 & -208.0 & 212.7 & -214.0 & 6 & ESO $1.5 \mathrm{~m}+\mathrm{B} \& \mathrm{C}$ \\
\hline 206.852 & 0.022 & -11.6 & -11.6 & -56.3 & 38.1 & 6 & $\mathrm{ESO} 1.5 \mathrm{~m}+\mathrm{B} \& \mathrm{C}$ \\
\hline 207.816 & 0.188 & -207.2 & 203.6 & -200.8 & 204.2 & 6 & $\mathrm{ESO} 1.5 \mathrm{~m}+\mathrm{B} \& \mathrm{C}$ \\
\hline 208.795 & 0.356 & -156.1 & 155.8 & -166.8 & 164.4 & 6 & $\mathrm{ESO} 1.5 \mathrm{~m}+\mathrm{B} \& \mathrm{C}$ \\
\hline 208.879 & 0.370 & -150.4 & 136.7 & -148.9 & 164.1 & 6 & $\mathrm{ESO} 1.5 \mathrm{~m}+\mathrm{B} \& \mathrm{C}$ \\
\hline 209.789 & 0.527 & -7.5 & -7.5 & 1.7 & 1.7 & 6 & $\mathrm{ESO} 1.5 \mathrm{~m}+\mathrm{B} \& \mathrm{C}$ \\
\hline 209.903 & 0.546 & -7.0 & -7.0 & -10.4 & -8.6 & 6 & $\mathrm{ESO} 1.5 \mathrm{~m}+\mathrm{B} \& \mathrm{C}$ \\
\hline 210.782 & 0.698 & 167.3 & -173.9 & 184.7 & -167.2 & 6 & $\mathrm{ESO} 1.5 \mathrm{~m}+\mathrm{B} \& \mathrm{C}$ \\
\hline 210.910 & 0.720 & 187.2 & -196.4 & 183.8 & -199.2 & 6 & $\mathrm{ESO} 1.5 \mathrm{~m}+\mathrm{B} \& \mathrm{C}$ \\
\hline 211.777 & 0.869 & 186.7 & -187.8 & 203.2 & -180.6 & 6 & ESO $1.5 \mathrm{~m}+\mathrm{B} \& \mathrm{C}$ \\
\hline 211.912 & 0.892 & 175.0 & -177.9 & 194.8 & -178.9 & 6 & $\mathrm{ESO} 1.5 \mathrm{~m}+\mathrm{B} \& \mathrm{C}$ \\
\hline 534.902 & 0.426 & -75.7 & 129.5 & -110.3 & 95.9 & 6 & $\mathrm{ESO} 1.5 \mathrm{~m}+\mathrm{B} \& \mathrm{C}$ \\
\hline 535.904 & 0.599 & 70.7 & -110.5 & 91.1 & -114.9 & 6 & $\mathrm{ESO} 1.5 \mathrm{~m}+\mathrm{B} \& \mathrm{C}$ \\
\hline 536.888 & 0.768 & 213.2 & -208.9 & 209.1 & -210.1 & 6 & $\mathrm{ESO} 1.5 \mathrm{~m}+\mathrm{B} \& \mathrm{C}$ \\
\hline 537.897 & 0.941 & 98.7 & -73.8 & 116.9 & -121.8 & 6 & $\mathrm{ESO} 1.5 \mathrm{~m}+\mathrm{B} \& \mathrm{C}$ \\
\hline 538.889 & 0.112 & -165.6 & 135.6 & -163.5 & 156.3 & 6 & $\mathrm{ESO} 1.5 \mathrm{~m}+\mathrm{B} \& \mathrm{C}$ \\
\hline 539.907 & 0.287 & -201.4 & 191.8 & -200.0 & 191.5 & 6 & $\mathrm{ESO} 1.5 \mathrm{~m}+\mathrm{B} \& \mathrm{C}$ \\
\hline 531.908 & 0.912 & 160.2 & -155.2 & 160.2 & -155.2 & 1 & $\mathrm{CAT}+\mathrm{CES}+\mathrm{LC}$ \\
\hline 532.881 & 0.079 & -97.9 & 122.7 & -97.9 & 122.7 & 1 & $\mathrm{CAT}+\mathrm{CES}+\mathrm{LC}$ \\
\hline 533.859 & 0.247 & -206.7 & 219.7 & -206.7 & 219.7 & 1 & $\mathrm{CAT}+\mathrm{CES}+\mathrm{LC}$ \\
\hline 534.844 & 0.416 & -99.7 & 108.2 & -99.7 & 108.2 & 1 & $\mathrm{CAT}+\mathrm{CES}+\mathrm{LC}$ \\
\hline 535.859 & 0.591 & 48.7 & -85.6 & 48.7 & -85.6 & 1 & $\mathrm{CAT}+\mathrm{CES}+\mathrm{LC}$ \\
\hline 536.856 & 0.762 & 219.0 & -201.2 & 219.0 & -201.2 & 1 & $\mathrm{CAT}+\mathrm{CES}+\mathrm{LC}$ \\
\hline 622.604 & 0.506 & -4.9 & 1.9 & -4.9 & 1.9 & 1 & $\mathrm{CAT}+\mathrm{CES}+\mathrm{LC}$ \\
\hline 623.628 & 0.682 & 154.2 & -170.8 & 154.2 & -170.8 & 1 & $\mathrm{CAT}+\mathrm{CES}+\mathrm{LC}$ \\
\hline 624.554 & 0.841 & 215.8 & -211.1 & 215.8 & -211.1 & 1 & $\mathrm{CAT}+\mathrm{CES}+\mathrm{LC}$ \\
\hline 625.607 & 0.022 & -2.2 & -2.2 & -2.2 & -2.2 & 1 & $\mathrm{CAT}+\mathrm{CES}+\mathrm{LC}$ \\
\hline 626.571 & 0.188 & -202.0 & 214.9 & -202.0 & 214.9 & 1 & $\mathrm{CAT}+\mathrm{CES}+\mathrm{LC}$ \\
\hline 1000.668 & 0.509 & 0.3 & 3.1 & 0.3 & 3.1 & 1 & $\mathrm{CAT}+\mathrm{CES}+\mathrm{VLC}$ \\
\hline 1299.817 & 0.945 & 136.7 & -91.8 & 130.4 & -103.7 & 11 & ESO $1.5 \mathrm{~m}+$ FEROS \\
\hline 1300.809 & 0.115 & -147.8 & 163.4 & -150.5 & 166.8 & 11 & ESO $1.5 \mathrm{~m}+$ FEROS \\
\hline 1301.815 & 0.288 & -204.3 & 202.5 & -202.4 & 194.7 & 11 & ESO $1.5 \mathrm{~m}+$ FEROS \\
\hline 1302.808 & 0.459 & -12.9 & -12.9 & -50.1 & 42.6 & 11 & ESO $1.5 \mathrm{~m}+$ FEROS \\
\hline 1304.889 & 0.817 & 223.2 & -213.0 & 213.0 & -210.3 & 11 & ESO $1.5 \mathrm{~m}+$ FEROS \\
\hline 1323.740 & 0.058 & -88.6 & 49.4 & -86.8 & 76.5 & 11 & ESO $1.5 \mathrm{~m}+$ FEROS \\
\hline 1327.780 & 0.753 & 199.2 & -202.4 & 200.7 & -202.9 & 11 & ESO $1.5 \mathrm{~m}+$ FEROS \\
\hline 1328.734 & 0.917 & 151.8 & -150.2 & 158.2 & -144.5 & 6 & CTIO $1.5 \mathrm{~m}+\mathrm{BME}$ \\
\hline 1329.738 & 0.089 & -130.2 & 117.9 & -122.6 & 136.2 & 6 & CTIO $1.5 \mathrm{~m}+\mathrm{BME}$ \\
\hline 1330.739 & 0.261 & -215.9 & 194.0 & -212.9 & 184.1 & 6 & CTIO $1.5 \mathrm{~m}+\mathrm{BME}$ \\
\hline 1331.771 & 0.439 & -81.4 & 36.7 & -91.5 & 60.6 & 6 & CTIO $1.5 \mathrm{~m}+\mathrm{BME}$ \\
\hline 1332.798 & 0.615 & 84.8 & -108.4 & 94.7 & -107.2 & 6 & CTIO $1.5 \mathrm{~m}+\mathrm{BME}$ \\
\hline 1668.832 & 0.393 & -108.1 & 131.5 & -120.5 & 125.2 & 11 & ESO $1.5 \mathrm{~m}+$ FEROS \\
\hline 1669.853 & 0.568 & 36.0 & -42.3 & 37.2 & -51.4 & 11 & ESO $1.5 \mathrm{~m}+$ FEROS \\
\hline 1670.836 & 0.737 & 206.7 & -190.0 & 204.2 & -188.4 & 11 & ESO $1.5 \mathrm{~m}+$ FEROS \\
\hline 1671.839 & 0.910 & 157.9 & -158.6 & 163.2 & -159.3 & 11 & ESO $1.5 \mathrm{~m}+\mathrm{FEROS}$ \\
\hline 1672.828 & 0.080 & -122.7 & 102.1 & -120.9 & 111.4 & 11 & ESO $1.5 \mathrm{~m}+$ FEROS \\
\hline 1673.809 & 0.248 & -203.6 & 217.2 & -206.4 & 207.6 & 11 & ESO $1.5 \mathrm{~m}+$ FEROS \\
\hline
\end{tabular}

exposure times were of the order of $20 \mathrm{~min}$ and the average $\mathrm{S} / \mathrm{N}$ ratio is above 150 . The wavelength domain is centered on the He I $\lambda 4471, \mathrm{~N}$ III $\lambda 4641$ or He II $\lambda 4686$ lines and is $\sim 40 \AA$ and $\sim 20 \AA$ wide for spectra taken with the $\mathrm{LC}$ and the VLC respectively.
During three observing runs in April - May 1999, end of May 1999 and May 2000, a set of echelle spectra was taken with the Fiber-fed Extended Range Optical Spectrograph (FEROS) attached to the ESO $1.5 \mathrm{~m}$ telescope at La Silla. Thirty seven orders corresponding to a 
wavelength domain from 3650 to $9200 \AA$ were observed. Typical exposure times were of the order of $6 \mathrm{~min}$ and the average $\mathrm{S} / \mathrm{N}$ ratio is about 100, 220 and 150 at 4050, 6000 and $7500 \AA$ respectively. The spectral resolving power of the FEROS instrument is 48000 . The detector was an EEV CCD with $2048 \times 4096$ pixels of $15 \times 15 \mu \mathrm{m}$.

Finally, another set of echelle spectra over the range 3850 to $5790 \AA$ was obtained with the Bench-Mounted Echelle Spectrograph (BME) attached to the $1.5 \mathrm{~m}$ CTIO Ritchey-Chrétien Telescope, during a 5 night run in June 1999. Forty nine orders were observed using the KPGL2 316 lines $\mathrm{mm}^{-1}$ grating as a cross-disperser. The detector was a Tek 2048 CCD with $24 \mu \mathrm{m}$ pixels. The slit width was set to $70 \mu \mathrm{m}$ corresponding to a resolving power of 45 000. Exposure times were of the order of $45 \mathrm{~min}$ and the average $\mathrm{S} / \mathrm{N}$ ratio in the continuum is about 100 .

\subsection{Data reduction}

The B\&C and CAT observations were reduced in a standard way using the MIDAS package provided by ESO. Whenever possible, the spectra were rectified using a reference star observed under the same conditions. When such a spectrum was not available the spectra were normalized by fitting a polynomial to the continuum.

We used the FEROS context working under the MIDAS environment to reduce the FEROS echelle spectra. The reduction was performed using the standard option. Most of the unavoidable strong fringes that affect the red part of the spectrum (above $6600 \AA$ ) are simply corrected by flat-fielding. There are however a few residual fringes near $6670,7140,7385$ and $7930 \AA$. Finally, the spectra were normalized over a range of a few hundred $\AA$ at once by fitting a polynomial of degree 4 or 5 to the continuum. Whenever doubt existed about the quality of the automatic merging between the different orders, we checked our results using the unmerged spectrum. Good agreement between both methods was generally observed.

The BME data were reduced using the IRAF ${ }^{1}$ package and following the recommendations of the BME User's Manual. The pixel to pixel variations were removed using flat field exposures taken with a very bright light source and a diffusing screen placed inside the spectrograph (socalled milky flats). A first rectification of the extracted echelle orders was carried out with the projector flat exposures. The spectra were then normalized by fitting a low-order polynomial to the continuum.

\section{4. $R V$ measurements and orbital solutions}

The inspection of our data reveals that the spectra of the primary and secondary are very similar and that every absorption line detected is present in both spectra. Several lines are further present in emission and we will discuss them later in this paper.

${ }^{1}$ IRAF is distributed by the National Optical Astronomy Observatories.

\subsection{RV measurements}

We selected eleven pure absorption lines in the spectrum of HD 152248 to compute the orbital elements of the system. These lines were chosen according to the following criteria: the intensity of the line, the fact that they do not suffer a heavy blend with another neighbouring line at large $R V$ separation phases (e.g. $\mathrm{H} \delta, \mathrm{C}$ IV $\lambda \lambda 5801-12$ ) and the requirement that they should be free from pollution by any ISM lines (e.g. H $\epsilon$ ) or telluric lines (e.g. He I $\lambda 7065)$. The selected lines are listed in Table 2. We measured the Doppler shifts by fitting two Gaussians at phases where the separation between the lines of both stars was sufficient. We then used the cross-correlation like method described in Rauw et al. (2000) to attempt to disentangle the blended lines. As HD 152248 is an eclipsing binary, we used the light curve of PGB to achieve a rough first order correction of the relative line intensity at eclipsing phases. The observed lines are further affected by slight intensity and/or profile variations; we assumed, from our experience, that the errors on Doppler shifts measured in this way are about 2.5 times larger than the errors on the two-Gaussian fit results. For those phases where the lines were too heavily blended for the latter method to give reliable results, we adopted the $R V$ obtained with a single Gaussian fit. We estimated, from the $F W H M$ of the blend, that the accuracy on the line position in this latter case could be ten times lower than the one reached at large separation phases (i.e. with the two-Gaussian fit method). While computing the orbital solution, we thus attributed a relative weight of $1.0,0.15$ and 0.01 to the $R V \mathrm{~s}$ respectively measured with the two-Gaussian fit, the cross-correlation like and the single Gaussian fit methods. Ruling out the single Gaussian fit points provides orbital parameters that are only marginally different.

We adopted the effective wavelengths for O stars listed in Table 2 to compute the barycentric $R V \mathrm{~s}$. These effective wavelengths are from Conti et al. (1977) below $5000 \AA$, and from Underhill (1994) above. As our spectra taken with different instruments cover different wavelength ranges, the number of $R V$ points associated with the different lines is variable. We report this number in Col. 6 of Table 2. The He I $\lambda 4471$ line provides the largest data set with $47 R V$ points. These measurements are listed in Table 1.

\subsection{Orbital solutions}

\subsubsection{The code}

Our orbital solution computation code is based on a modified version of the Wolfe, Horak \& Storer algorithm (see Wolfe et al. 1967, for the method; and Rauw et al. 2000, for the modifications implemented) and requires a previous knowledge of the orbital period to be run. 
Table 2. List of the absorption lines used to compute the orbital solution together with the adopted effective wavelengths. Column 3 gives the best-fit period value as deduced from the different $R V$ data sets. The errors quoted were computed using the Wolfe, Horak \& Storer algorithm. The primary and secondary apparent systemic velocities derived are respectively listed in Cols. 4 and 5. The last column provides the number of $R V$ points associated with each line data set

\begin{tabular}{|c|c|c|c|c|c|}
\hline Line & $\begin{array}{c}\text { Effective } \\
\text { wavelength }(\AA)\end{array}$ & Period (days) & $\gamma_{\lambda, 1}\left(\mathrm{~km} \mathrm{~s}^{-1}\right)$ & $\gamma_{\lambda, 2}\left(\mathrm{~km} \mathrm{~s}^{-1}\right)$ & $n$ \\
\hline He I $\lambda 4026$ & 4026.072 & $5.816072 \pm 0.000196$ & $-34.1 \pm 3.7$ & $-36.0 \pm 8.1$ & 30 \\
\hline He II $\lambda 4200$ & 4199.830 & $5.815943 \pm 0.000187$ & $-7.9 \pm 3.1$ & $-14.7 \pm 6.4$ & 35 \\
\hline $\mathrm{H} \gamma$ & 4340.468 & $5.816123 \pm 0.000222$ & $-43.6 \pm 3.9$ & $-36.3 \pm 8.8$ & 35 \\
\hline He I $\lambda 4471$ & 4471.512 & $5.816045 \pm 0.000092$ & $-30.5 \pm 1.5$ & $-28.5 \pm 4.3$ & 47 \\
\hline He II $\lambda 4542$ & 4541.590 & $5.816022 \pm 0.000116$ & $-24.4 \pm 2.0$ & $-18.8 \pm 4.9$ & 35 \\
\hline He I $\lambda 4713$ & 4713.200 & $5.815972 \pm 0.000224$ & $-29.1 \pm 4.3$ & $-20.7 \pm 9.6$ & 30 \\
\hline $\mathrm{H} \beta$ & 4861.190 & $5.816508 \pm 0.000849$ & $-37.2 \pm 5.0$ & $-37.7 \pm 11.7$ & 18 \\
\hline Не г $\lambda 4922$ & 4921.929 & $5.815834 \pm 0.000487$ & $-26.8 \pm 2.7$ & $-23.5 \pm 7.1$ & 13 \\
\hline He I $\lambda 5016$ & 5015.680 & $5.816139 \pm 0.000965$ & $-19.9 \pm 6.3$ & $-12.6 \pm 14.4$ & 13 \\
\hline He II $\lambda 5411$ & 5411.520 & $5.815877 \pm 0.000515$ & $-19.0 \pm 3.1$ & $-15.3 \pm 8.0$ & 13 \\
\hline O III $\lambda 5592$ & 5592.370 & $5.816510 \pm 0.000864$ & $-18.8 \pm 4.3$ & $-22.2 \pm 11.5$ & 18 \\
\hline
\end{tabular}

\subsubsection{The period}

The best (i.e. with the smallest rms error) estimate of the period from the literature is $P=5.816083 \pm 0.000019$ days as given by ST96. As the He I $\lambda 4471 R V$ s are the most numerous among our $R V$ data, we first used this set to investigate the period value. For this purpose we applied the Lafler \& Kinman method (Lafler \& Kinman 1965) and the generalized spectrogramme Fourier technique (Heck et al. 1985). The results obtained with both methods lie within $510^{-4}$ days of the value of ST96, which is one order of magnitude smaller than the error estimated from the width of the peak in the periodogramme. We then iteratively ran our code, allowing for a differential correction of the orbital period. The convergence was reached in each case after a few iterations at most. This provides us with the period value which yields the orbital solution that best matches the observations (i.e. with the lowest rms residual) of the considered data set. Column 3 of Table 2 lists the period values obtained. Their weighted mean is $P=5.816032 \pm 0.000058$ days, which is very close to the He I $\lambda 4471$ value and contains both ST96 and Mayer et al. (1992) values within a $\pm 1 \sigma$ interval. The very small uncertainty of the ST96 period determination results from the large time base of their IUE data set ( $\sim 5860$ days) which is almost exactly four times ours. However, the HD 152248 system is most probably undergoing an apsidal motion of a few degrees per year (see below and Mayer et al. 2001) and the large time base of ST96 could actually bias their period determination. In the following, we choose to adopt our mean value $P=5.816032$ days for the orbital period.

\subsubsection{The orbital solutions}

As a first step, we computed an orbital solution for each of the 11 absorption line data sets. Accounting for the error bars, the computed orbital elements do agree with each other, except for the apparent systemic velocities $\gamma_{1}$ and $\gamma_{2}$ (respectively associated with the primary and secondary star). Though the $\gamma_{1}$ and $\gamma_{2}$ values are in acceptable agreement with each other for the same line (see Table 2), their values as deduced from the different lines are indeed significantly different. This illustrates the well-known effect that different lines might reflect different systemic velocities. This might stem from the fact that these lines are formed at different depths in the atmosphere and thus at different outward velocities, resulting in slightly different apparent systemic velocities.

In order to combine the $R V \mathrm{~s}$ obtained from the different lines, we had to refer all the $R V$ measurements to a "zero systemic velocity" reference frame. For this purpose, we simply subtracted the corresponding weighted mean between $\gamma_{1}$ and $\gamma_{2}$ from the individual $R V$ s of the considered line. We then computed a weighted $R V$ mean from all the $R V$ data obtained at the same observing date. These mean $R V \mathrm{~s}$ in the zero systemic velocity reference frame are listed in Cols. 5 and 6 of Table 1.

The computed orbital solutions from the He I $\lambda 4471$ $R V$ and the mean $R V$ sets are both presented in Table 3. These two solutions are in excellent agreement with each other. Our way of combining the $R V \mathrm{~s}$ from the different lines is further justified by the fact that the apparent systemic velocities computed from the mean data lie well within $1 \sigma$ of the zero velocity. We also tested the value of the period found by ST96. This does not affect the rms residual by more than a few tenths of $\mathrm{kms}^{-1}$ and the difference between the computed orbital elements is not significant.

\subsection{Physical parameters}

The He I $\lambda 4471$ velocity curve is plotted in Fig. 1. This solution deduced from the optical wavelength domain is in good general agreement with the previous determination by ST96 from the UV domain. Our apparent systemic velocity is however $12 \mathrm{~km} \mathrm{~s}^{-1}$ more positive than theirs. This difference might result from the zero velocity point in their cross-correlation method, but it is probable too that the optical and the UV domains reflect different 
Table 3. Computed orbital solution for HD 152248 using the $R V \mathrm{~s}$ of the He I $\lambda 4471$ absorption lines (left) or the mean $R V \mathrm{~s}$ (right). $T_{0}$ is the time of the periastron passage

\begin{tabular}{lrlrl}
\hline & $\begin{array}{r}\text { He I } \lambda 4471 \\
\text { common solution }\end{array}$ & $\begin{array}{r}\text { Mean } R V \mathrm{~s} \\
\text { common solution }\end{array}$ \\
\hline$P($ days $)$ & 0.127 & \pm 0.007 & 0.134 & \pm 0.006 \\
$e$ & 84.8 & \pm 4.7 & 81.8 & \pm 4.3 \\
$\omega\left(^{\circ}\right)$ & 2003.879 & \pm 0.072 & 2003.841 & \pm 0.066 \\
$T_{0}(\mathrm{HJD}$ & & & & \\
$-2450000)$ & -30.3 & \pm 1.5 & 0.2 & \pm 1.4 \\
$\gamma_{1}\left(\mathrm{~km} \mathrm{~s}^{-1}\right)$ & 216.0 & \pm 1.5 & 216.6 & \pm 1.3 \\
$K_{1}\left(\mathrm{~km} \mathrm{~s}^{-1}\right)$ & 24.59 & \pm 0.17 & 24.66 & \pm 0.15 \\
$a_{1} \sin i\left(R_{\odot}\right)$ & -28.7 & \pm 4.3 & 2.6 & \pm 3.4 \\
$\gamma_{2}\left(\mathrm{~km} \mathrm{~s}^{-1}\right)$ & 213.7 & \pm 5.2 & 214.3 & \pm 4.2 \\
$K_{2}\left(\mathrm{~km} \mathrm{~s}^{-1}\right)$ & 24.35 & \pm 0.62 & 24.40 & \pm 0.50 \\
$a_{2} \sin i\left(R_{\odot}\right)$ & 9.5 & & 8.2 & \\
$\mathrm{rms} \mathrm{residual} \quad\left(\mathrm{km} \mathrm{s}^{-1}\right)$ & 0.990 & \pm 0.023 & 0.990 & \pm 0.018 \\
$q\left(=m_{1} / m_{2}\right)$ & 23.19 & \pm 1.19 & 23.34 & \pm 0.96 \\
$m_{1} \sin ^{3} i\left(M_{\odot}\right)$ & 23.44 & \pm 0.73 & 23.58 & \pm 0.61 \\
$m_{2} \sin ^{3} i\left(M_{\odot}\right)$ & & & &
\end{tabular}



Fig. 1. Radial velocity curve of the HD 152248 binary system as computed from the He I $\lambda 4471$ line plotted against the phase $(\phi)$. Different symbols refer to different instruments: triangle $=$ $\mathrm{BME}$, square $=\mathrm{B} \& \mathrm{C}$, diamond $=\mathrm{CES}$, circle $=$ FEROS. Different sizes indicate different weights assigned to the data points in the computation of the orbital solution. Open symbols stand for the primary, while filled symbols indicate the secondary's $R V \mathrm{~s}$

apparent systemic velocities. Though the primary parameters from both our and their solution are very close, a more intriguing discrepancy is the difference between the mass ratios deduced. Indeed, our mass ratio $(q=0.990)$ is much closer to unity than the ST96 value $(q=0.941)$, and this manifests in the fact that our secondary component parameters $K_{2}$ and $a_{2} \sin i$ are larger. The minimal masses we derive are also larger than the values inferred by ST96 and PGB. Adopting the PGB value for the inclination $i=72^{\circ}$ yields absolute masses of $M_{1}=27.0 M_{\odot}$ and $M_{2}=27.2 M_{\odot}$ for the primary and the secondary respectively. We used Eggleton's formula (Eggleton 1983) to estimate the radii of the Roche lobe $(R R L)$ for both components. Again assuming $i=72^{\circ}$ yields respectively $R R L_{1}=19.45 R_{\odot}$ and $R R L_{2}=19.55 R_{\odot}$ if we neglect the effect of the eccentricity (however see below). Thus if we adopt $R_{1}=13.4 R_{\odot}$ and $R_{2}=12.9 R_{\odot}$, this result confirms the assertion of PGB that the system should not be undergoing a Roche lobe overflow (RLOF) mass transfer. However, Mayer et al. (2001) reported a lower value for the inclination $\left(i=67.2^{\circ}\right)$ and larger radii $\left(R_{1}=15.6 R_{\odot}\right.$ and $\left.R_{2}=17.1 R_{\odot}\right)$. In this latter case, the primary and secondary are respectively filling 72 and $91 \%$ of their Roche lobe volume at periastron. If these preliminary results of Mayer et al. (2001) for the radii are confirmed, we then expect the system to be very unstable near periastron passage and important mass transfer or mass loss could be initiated.

\subsection{Apsidal motion}

While recomputing the orbital solution from previously published $R V$ data at different epochs in order to check the consistency of our method, we were led to suspect the presence of an apsidal motion within the binary system HD 152248. In the following investigation, we considered three sets of data that are summarized in Table 4 . We decided to ignore the complementary data obtained by HCB near JD $\sim 2440000$ because their very poor phase coverage did not allow us to derive any significant constraint on the longitude of periastron. Keeping the value of the orbital period and the eccentricity fixed at our new value, we have fitted the $R V$ data from the literature. Table 4 lists the $\omega$ values that provide the best fit to the various data sets. From these results, it is clear that the system is undergoing an apsidal motion. A simple linear regression yields a rate for the apsidal motion of about $3.4^{\circ} \mathrm{yr}^{-1}$. A more detailed study by Mayer et al. (2001) based on new recent photometric results is currently underway. These authors independently discovered the presence of an apsidal motion in HD 152248 and they estimate a period of 132 years, in acceptable agreement with our value.

\section{Equivalent widths and S-S effect}

Using a two-Gaussian fit, we measured the $E W$ s of the primary and secondary absorption lines listed in Table 2, except near conjunction. Particular attention has been paid to the normalization process to avoid systematic deviations. We estimate that our continuum determination is self-consistent with an accuracy better than $0.5 \%$. For the strongest lines, this corresponds to a maximum contribution to the error on the $E W$ measurements of about $0.05 \AA$. We further estimate that the mean contribution of the continuum determination to the error is about $0.02 \AA$ 
Table 4. Summary of the investigation we undertook to estimate the apsidal motion of the HD 152248 system. Second to fourth columns list respectively the approximative mean JD in format JD -2400000 , the time interval (expressed in days) and the number of $R V$ points in the set. Column 5 reports the values of the angle of periastron passage $(\omega)$ which provide the best fit. The last column lists the rms residual between the best fit and the data

\begin{tabular}{cccccc}
\hline Data set & $\overline{\mathrm{JD}}$ & $\begin{array}{c}\text { Time } \\
\text { base }\end{array}$ & $\mathrm{n}$ & $\omega\left(^{\circ}\right)$ & $\begin{array}{c}\mathrm{rms} \\
\left(\mathrm{km} \mathrm{s}^{-1}\right)\end{array}$ \\
\hline Struve $^{1}$ & $\sim 31200$ & 14 & 22 & -95 & 27.5 \\
ST96 & $\sim 48500$ & 5861 & 26 & 76 & 12.1 \\
This work & $\sim 51000$ & 1468 & 47 & 85 & 9.2 \\
\hline
\end{tabular}

1. Struve (1944); also described in HCB.
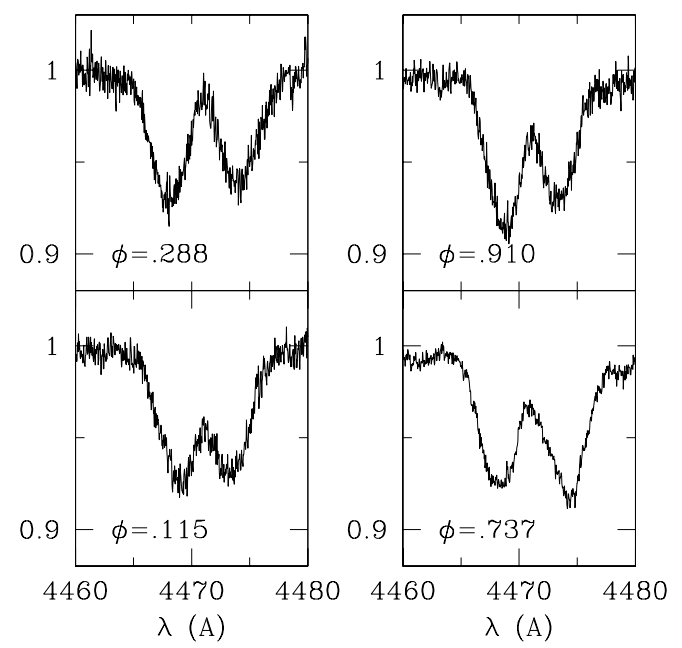

Fig. 2. Illustration of the variation of the He I $\lambda 4471$ line strength. Note that the primary is on the blue side of the spectra at phases $\phi=0.115$ and 0.288 while it is on the red side of the spectra at the phases shown in the right panel. The depletion in the red wing of the spectrum at $\phi=0.737$ is the blue shifted secondary Mg II $\lambda 4481$ line
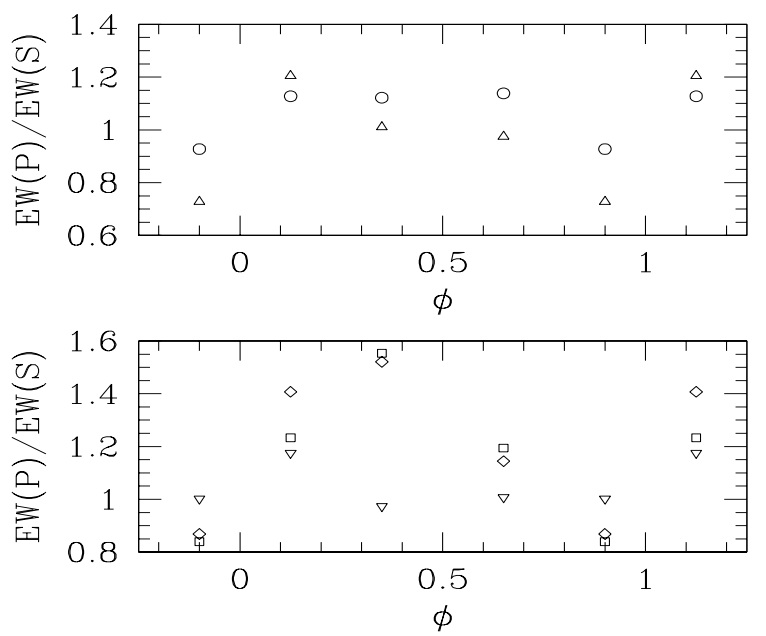

Fig. 3. Primary to secondary ratios of the mean $E W \mathrm{~s}$ of the He I lines in each quadrant. Downwards triangles $=$ He I $\lambda 4026$, circles $=$ He I $\lambda 4471$, diamonds $=$ He I $\lambda 4713$, squares $=$ He I $\lambda 4922$, upright triangles $=$ He I $\lambda 5016$
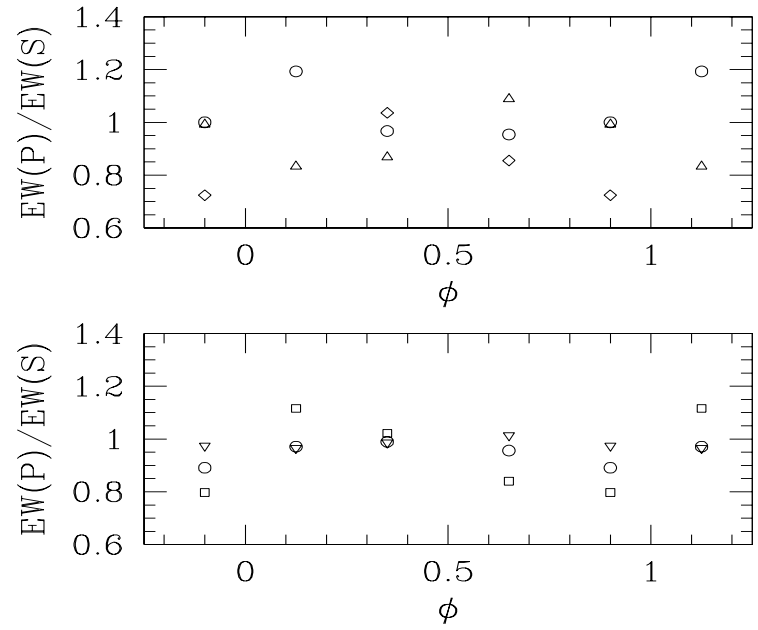

Fig. 4. Primary to secondary ratios of the mean $E W \mathrm{~s}$ of the Balmer and the O III (upper panel) lines and of the He II (lower panel) lines in each quadrant. Upper panel: circles $=\mathrm{H} \gamma$, diamonds $=\mathrm{H} \beta$, upright triangles $=\mathrm{O}$ III $\lambda 5592$. Lower panel: squares $=$ He II $\lambda 4200$, circles $=$ He II $\lambda 4542$, downwards triangles $=$ HeII $\lambda 5411$

and that the intrinsic error related to the measurement process is of the same order of magnitude.

Several clear tendencies appear from these measurements and are presented below. First of all, let us divide the orbital cycle into four parts which we call quadrants, each of them corresponding to an interval of $90^{\circ}$ in true anomaly. The first quadrant ranges then from the primary eclipse to the first quadrature; the second, from the first quadrature to the secondary eclipse and so on for the third and fourth quadrants. Due to the blend near conjunction, the effective corresponding phase intervals are, respectively for 1 st to 4 th interval, [0.06-0.22], [0.22-0.40], [0.66-0.80] and [0.80-0.95]. To limit the uncertainties and outline the observed trends, we computed the mean $E W \mathrm{~s}$ over each of the quadrants. These are listed in Table 5. The primary to secondary ratios of the mean $E W_{\mathrm{s}}$ in each quadrant are further plotted in Figs. 3 and 4.

From these figures, we can identify the following trends:

1. The He I lines: a very intriguing variation is observed for the He I $\lambda 4471$ line and is also present in other He I lines. The primary to secondary $E W$ ratio is reversed in the fourth quadrant. Indeed, during the first three quadrants, the lines associated with the primary are slightly more intense than the secondary ones. In the fourth quadrant (i.e. $\phi=0.8-1.0$ ) however the situation is exactly the opposite, with the primary's lines being fainter. Thus the secondary lines seem stronger between phases 0.8 and 1.0, when the star is approaching. However, we have to mention that this effect is not detected for the He I $\lambda 4026$ line, but this line is actually a blend with the He II $\lambda 4026$ transition;

2. The He II lines: a clear enhancement of the secondary lines compared to the primary ones is observed for the He II $\lambda \lambda 4200$ and 4542 lines during the 3rd and 
Table 5. List of the mean equivalent widths (expressed in $\AA$ ) over each of the four quadrants (see text). The P and S stand for Primary and Secondary respectively

\begin{tabular}{ccccccccc}
\hline \multirow{2}{*}{ Line } & \multicolumn{2}{c}{ Quadrant 1 } & \multicolumn{2}{c}{ Quadrant 2 } & \multicolumn{2}{c}{ Quadrant 3 } & \multicolumn{2}{c}{ Quadrant 4 } \\
& $\mathrm{P}$ & $\mathrm{S}$ & $\mathrm{P}$ & $\mathrm{S}$ & $\mathrm{P}$ & $\mathrm{S}$ & $\mathrm{P}$ & $\mathrm{S}$ \\
\hline He I $\lambda 4026$ & 0.281 & 0.239 & 0.242 & 0.248 & 0.258 & 0.256 & 0.265 & 0.264 \\
He I $\lambda 4471$ & 0.299 & 0.265 & 0.296 & 0.264 & 0.302 & 0.265 & 0.266 & 0.287 \\
He I $\lambda 4713$ & 0.095 & 0.068 & 0.094 & 0.062 & 0.086 & 0.075 & 0.063 & 0.073 \\
He I $\lambda 4922$ & 0.101 & 0.082 & 0.116 & 0.075 & 0.107 & 0.090 & 0.087 & 0.104 \\
He I $\lambda 5016$ & 0.106 & 0.088 & 0.097 & 0.096 & 0.098 & 0.101 & 0.083 & 0.114 \\
\hline Hе II $\lambda 4200$ & 0.188 & 0.168 & 0.173 & 0.169 & 0.195 & 0.233 & 0.185 & 0.232 \\
Hе II $\lambda 4542$ & 0.280 & 0.288 & 0.283 & 0.286 & 0.283 & 0.296 & 0.268 & 0.301 \\
Hе I $\lambda 5411$ & 0.421 & 0.436 & 0.425 & 0.431 & 0.439 & 0.433 & 0.418 & 0.429 \\
\hline H $\gamma$ & 0.914 & 0.766 & 0.804 & 0.832 & 0.812 & 0.851 & 0.837 & 0.836 \\
H $\beta$ & $\mathrm{n}$ & $\mathrm{n}$. & 0.758 & 0.732 & 0.740 & 0.865 & 0.603 & 0.833 \\
О III $\lambda 5592$ & 0.195 & 0.234 & 0.260 & 0.299 & 0.203 & 0.186 & 0.191 & 0.193 \\
\hline
\end{tabular}

4th quadrants, while the secondary is approaching. However the He II $\lambda 5411$ line does not seem to display any significant variation of the $E W$ ratio (Fig. 4 );

3. The Balmer lines: these lines do not seem to display such a clear general trend as the He I or He II lines. Indeed, although the four Balmer absorptions (i.e. $\mathrm{H} \beta$, $\mathrm{H} \gamma, \mathrm{H} \delta$ and $\mathrm{H} \epsilon$ ) most often exhibit the blueshifted component with the largest $E W$, this probably results, for the $\mathrm{H} \delta$ and $\mathrm{H} \epsilon$ lines, from a blend with neighbouring lines on the blue side. The $\mathrm{H} \gamma$ line displays an inversion of the primary and secondary $E W$ ratio between the 1st and 2nd quadrants. Finally, though none of the $\mathrm{H} \beta$ spectra could be separated in the 1st quadrant, the secondary line is clearly strengthened when this star is approaching (i.e. quadrants 3 and 4). The primary line also seems fainter at those phases;

4. The O III $\lambda 5592$ line: this line displays a behaviour opposite to the ones of the lines previously discussed. In this case, the secondary line is fainter in the 3rd and 4th quadrants, while the secondary star is approaching.

As suggested by Howarth et al. (1997), these variations might be related to the so-called Struve-Sahade effect (S$\mathrm{S}$ effect) though the origin of this effect is still not fully understood. First reported by Struve (1937), the StruveSahade effect is the apparent strengthening of the secondary spectrum of a hot binary when the secondary is approaching and the corresponding weakening of the lines when it is receding (Bagnuolo et al. 1999). We refer to the paper of Bagnuolo et al. for a recent review of the problem. In their paper, Bagnuolo et al. re-analysed three systems previously reported to display this effect and concluded that these "three classical massive binaries [...] have a different tale": localized heating by colliding winds for AO Cas, no Struve-Sahade effect for Plaskett's Star and a probable RLOF scenario for $29 \mathrm{CMa}$. So the StruveSahade effect is thus probably a similar manifestation of different physical phenomena and must then be investigated one case at a time.

Strictly speaking, only $\mathrm{H} \beta$ and two of the three studied He II lines display the S-S effect. Indeed, the variation of the He I line intensity does not match the "classical" definition of the S-S effect, as the strengthening of the secondary lines and the corresponding weakening of the primary ones occur between the 3rd and the 4th quadrants. A detailed investigation of the intrinsic variations of the $E W \mathrm{~s}$ related to each star would further require correcting them for the relative contribution of the primary and secondary fluxes to the continuum. However, we have to defer this task to future works as we do not have at our disposal, at this stage, the appropriate photometric information required to properly carry out this study.

Finally, Gayley (2001) has recently suggested that the S-S effect might be due to surface flows generated by the irradiation of the stellar surface by the companion. This could modify the rotational broadening of the lines and might break down the approaching/receding symmetry, resulting in shallower or deeper absorption lines according to the line of sight. However, no influence on the intensity of the line is expected due to surface flows. The maximum projected velocity of such surface flows predicted in the case of the components of HD 152248 lies near $110 \mathrm{~km} \mathrm{~s}^{-1}$. The measured FWHMs of the absorption lines in the spectrum of HD 152248 present a scatter of about $0.3-0.4 \AA$ around the mean value, though no clear correlation with the phase is observed. This corresponds to a variation of the broadening velocity of about $25 \mathrm{~km} \mathrm{~s}^{-1}$. The exact contribution of surface flows induced by irradiation to these slight profile variations is unknown. Clearly, detailed numerical modelling as well as more observations combining very high $\mathrm{S} / \mathrm{N}$ and high resolution are needed to clarify this question.

\section{The emission lines}

As mentioned earlier in this paper, the HD 152248 spectrum presents several emission features. These include the He II $\lambda 4686$ and $\mathrm{H} \alpha$ lines that consist of a mixture of absorption and emission lines, the heavily blended $\mathrm{N}$ III $\lambda \lambda 4634-4641$ features, the C III $\lambda 5696$ line and the faint unidentified of emissions at $\lambda 4486$ and $\lambda 4504$. 

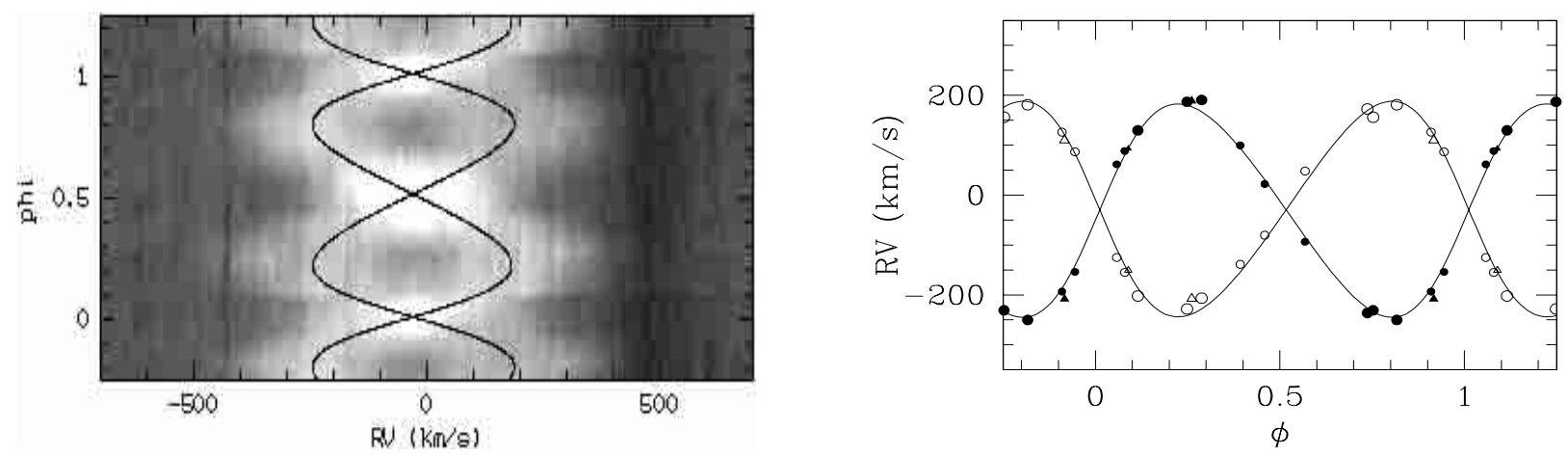

Fig. 5. Left: dynamical spectrum of the C III $\lambda 5696$ emission line. The cuts are set to [0.97-1.08]. The He I $\lambda 4471$ line $R V$ curve has been overplotted. Right: $R V \mathrm{~s}$ of the C III $\lambda 5696$ line overplotted on the He I $\lambda 4471$ line $R V$ curve. The symbols used have the same signification as in Fig. 1

\subsection{The CIII $\lambda 5696$ line}

As it clearly appears from Fig. 5 , the C III $\lambda 5696$ emission line turns out to be double peaked around quadrature and the peaks seem to move in phase with the $R V$ curves of the absorption lines. In order to measure the $R V \mathrm{~s}$ associated with these peaks, we first modelled the diffuse interstellar band (DIB) at $\lambda=5704.97 \AA(E W \approx 0.064 \AA)$ on a spectrum where it was well separated from the C III line (HJD 2451669.853). We then subtracted the modelled DIB from all spectra and fitted two Gaussians to the C III emission line whenever the separation between the components was large enough; at other phases, we used the cross-correlation like method. The measured $R V \mathrm{~s}$ are plotted together with the HeI $\lambda 4471 R V$ curve on the right panel of Fig. 5. The left panel presents a grey scale image around the $\mathrm{C}$ III line; the He I $\lambda 4471$ line orbital solution has been overplotted. It is clear from the two panels of Fig. 5 that the C III $\lambda 5696$ lines closely follow the orbital motion. As we already pointed out in the case of HD 93403 (Rauw et al. 2000), this is a strong argument in favour of a photospheric origin of the C III $\lambda 5696$ line as suggested by Nussbaumer (1971) and Cardona-Núnẽz (1978). Finally, unlike the case of HD 93403 (see Rauw et al. 2001), the C III $\lambda 5696$ line in the spectrum of HD 152248 does not seem to display a reversal of the intensity ratio due to the Struve-Sahade effect.

\subsection{The $N$ III $\lambda \lambda 4634-4641$ structure}

The HD 152248 spectrum presents a highly complex emission structure between about $\lambda=4625 \AA$ and $\lambda=4645 \AA$, which displays important phase-locked profile variations. In this region of the spectrum, the N III $\lambda \lambda 4634$ and 4641 lines are heavily blended with each other as well as with the neighbouring Si IV $\lambda 4631$ and C III $\lambda \lambda 4647-4650$ lines at all phases. Due to their lower resolution, we excluded the B\&C spectra from our study. Similarly we did not consider the CTIO spectra because of their relatively lower $\mathrm{S} / \mathrm{N}$ ratio. A complementary journal of the CAT NIII $\lambda \lambda 4634-4641$ observations is given in Table 6 . A careful comparison of the CAT and FEROS N III $\lambda \lambda 4634-4641$ spectra with the predicted line positions computed from our ephemerides and our orbital solution allows us to identify the components of the blend. Both stars of the HD 152248 system present the N III $\lambda \lambda 4634-4641$ lines in emission in their spectra. Following e.g. Mathys (1988), an (f) tag should then be added to their spectral classification. The Si IV $\lambda 4631$ and C III $\lambda \lambda 4647-4650$ lines are in absorption in both spectra.

\subsection{The unidentified $\lambda \lambda 4486,4504$ lines}

The two $\lambda \lambda 4486,4504$ emission lines are related to the Of phenomenon and still remain unidentified, although they have been known for several decades now. These two lines are rather faint in the spectra of HD 152248 and their peak intensities reach maxima of respectively $2.5 \%$ and $2 \%$ of the continuum level at conjunction phases. The stellar Mg II $\lambda 4481$ lines, the DIB near $\lambda 4502$ and the probable presence of the N III $\lambda \lambda 4510-4514$ absorption lines render this region of the spectrum very crowded and prevent us from performing a detailed analysis. It seems however clear that the $\lambda 4486$ line is double peaked with the two components closely following the orbital motion. The situation is less clear concerning the $\lambda 4504$ line, because of the DIB that affects the blue side of the line. A careful inspection of the spectra however suggests that the $\lambda 4504$ line might also be double peaked according to the orbital motion.

\subsection{The He II $\lambda 4686$ and $H \alpha$ lines}

We have a total of 51 He II $\lambda 4686$ line spectra (17 B\&C, 16 CES, 5 BME and 13 FEROS) at our disposal that provide a very good phase coverage. The journal of the CAT observations of He II $\lambda 4686$ is given in Table 6. Our $\mathrm{H} \alpha$ line data set is much more limited and consists of only 13 FEROS spectra. The normalization of the FEROS spectra around these two lines turned out to be a rather difficult task. Indeed, both lines are situated at the junction of two echelle orders and we had to normalize the spectra locally. To compare the B\&C spectra with the echelle spectra, we 
Table 6. Complementary journal of the CAT observations of the N III $\lambda \lambda 4634-4641$ and HeII $\lambda 4686$ lines. The notations used are identical to those of Table 1

\begin{tabular}{cccl}
\hline Line & HJD & $\phi$ & Instrument. \\
\hline N III $\lambda \lambda 4634-41$ & 621.781 & 0.364 & CAT + CES + LC \\
& 622.802 & 0.540 & CAT + CES + LC \\
623.805 & 0.712 & CAT + CES + LC \\
& 624.771 & 0.878 & CAT + CES + LC \\
& 625.785 & 0.053 & CAT + CES + LC \\
626.762 & 0.220 & CAT + CES + LC \\
\hline He II $\lambda 4686$ & 532.911 & 0.084 & CAT + CES + LC \\
& 533.902 & 0.254 & CAT + CES + LC \\
& 534.897 & 0.425 & CAT + CES + LC \\
& 535.895 & 0.597 & CAT + CES + LC \\
621.707 & 0.351 & CAT + CES + LC \\
622.735 & 0.528 & CAT + CES + LC \\
& 623.741 & 0.701 & CAT + CES + LC \\
624.700 & 0.866 & CAT + CES + LC \\
625.714 & 0.040 & CAT + CES + LC \\
& 626.696 & 0.209 & CAT + CES + LC \\
& 995.821 & 0.676 & CAT + CES + VLC \\
996.782 & 0.841 & CAT + CES + VLC \\
& 997.767 & 0.011 & CAT + CES + VLC \\
998.753 & 0.180 & CAT + CES + VLC \\
999.760 & 0.353 & CAT + CES + VLC \\
1000.727 & 0.520 & CAT + CES + VLC \\
\hline
\end{tabular}

Table 7. Measured equivalent widths of the three components of the He II $\lambda 4686$ and $\mathrm{H} \alpha$ lines as measured on the spectrum taken on HJD $2451673.809(\phi=0.248)$

\begin{tabular}{ccc}
\hline Line & $E W(\mathrm{He}$ II $\lambda 4686)$ & $E W(\mathrm{H} \alpha)$ \\
component & $(\AA)$ & $(\AA)$ \\
\hline Primary absorption & 0.245 & 0.590 \\
Secondary absorption & 0.178 & 0.400 \\
Emission & -0.630 & -2.588 \\
\hline
\end{tabular}

also had to apply a local normalization around He II $\lambda 4686$ on the $\mathrm{B} \& \mathrm{C}$ spectra, in order to account for the broad underlying emission extending from 4600 to $4720 \AA$.

Both the He II $\lambda 4686$ and $\mathrm{H} \alpha$ lines consist of a mixture of absorptions and emissions. Dynamical spectra of these two lines are displayed as a function of orbital phase on the grey-scale images in Fig. 7 . These were built by direct linear interpolation along the time axis of the observed spectra.

It is clear from these two diagrams and from Fig. 6 that the He II $\lambda 4686$ and $\mathrm{H} \alpha$ line profiles are strongly variable. For both lines, we can identify two absorption components at $R V \mathrm{~s}$ that closely match the orbital motion. These absorptions are thus most probably formed in the atmosphere of the stars of the system. These two absorption features are superimposed on a broader emission component. The width of this latter emission varies with orbital phase (see e.g. Fig. 6).

To quantify these variations, we first measured the total $E W$ of the lines. The results for both lines are plotted against the phase in Fig. 8. Concerning the He II $\lambda 4686$

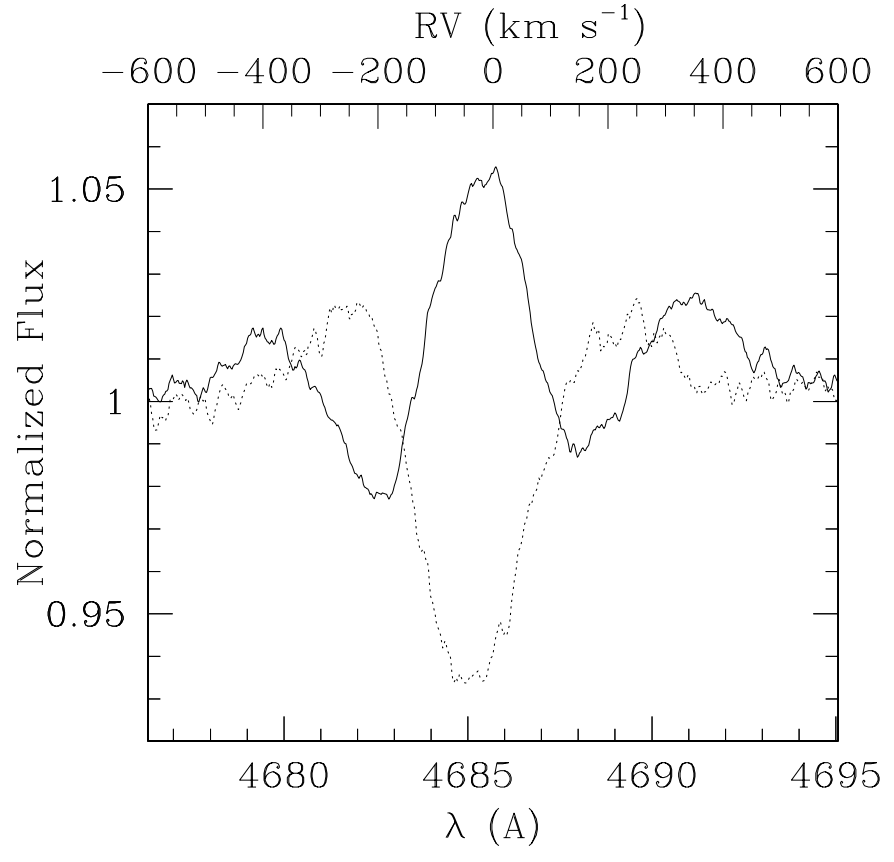

Fig. 6. Two superimposed spectra of the He II $\lambda 4686$ line: the full line corresponds to $\phi=0.910$; the dashed line, to $\phi=0.568$

line, we notice that the absorption is clearly dominating near phase $\phi=0.5$ and in a less prominent way near $\phi=0.0$. On the other side, the emission is overwhelming at phase $\phi \approx 0.8$ (and to some extent at $\phi \approx 0.25$ ).

We next attempted to restore the emission component. To this aim, in order to model the three line components, we fitted three Gaussians to the line profile on a spectrum where the two absorption contributions are well separated. The $E W \mathrm{~s}$ of all three components as obtained from the fit are reported in Table 7 . These results should however be considered with caution since the $E W$ s of the three components are most probably undergoing some variation with phase. For each observed spectrum, we then shifted the template of the absorption components according to our He I $\lambda 4471$ orbital solution and the observation phase, and we subtracted the shifted fake lines from the observed profile. This "restoration" process is a very crude method. In fact, the apparent systemic velocities of the He II $\lambda 4686$ and $\mathrm{H} \alpha$ absorption components are unknown and could be different from that of the He I $\lambda 4471$ line, though Fig. 7 suggests that this difference should be small. In addition, HD 152248 displays both ellipsoidal variations and photometric eclipses. As a consequence, the $E W \mathrm{~s}$ of the lines are most probably varying with phase, and it is almost impossible to correctly account for this effect without the exact knowledge of the amount of light originating from the primary and from the secondary at each orbital phase. However, we used the eclipse depths from PGB to derive a first order correction for the line intensities of the absorbing components. We then measured the full width at the base of the restored emission components. To this aim, we defined the limits of the emission at a normalized intensity of 1.01 , i.e. we considered the emission above $1 \%$ of 

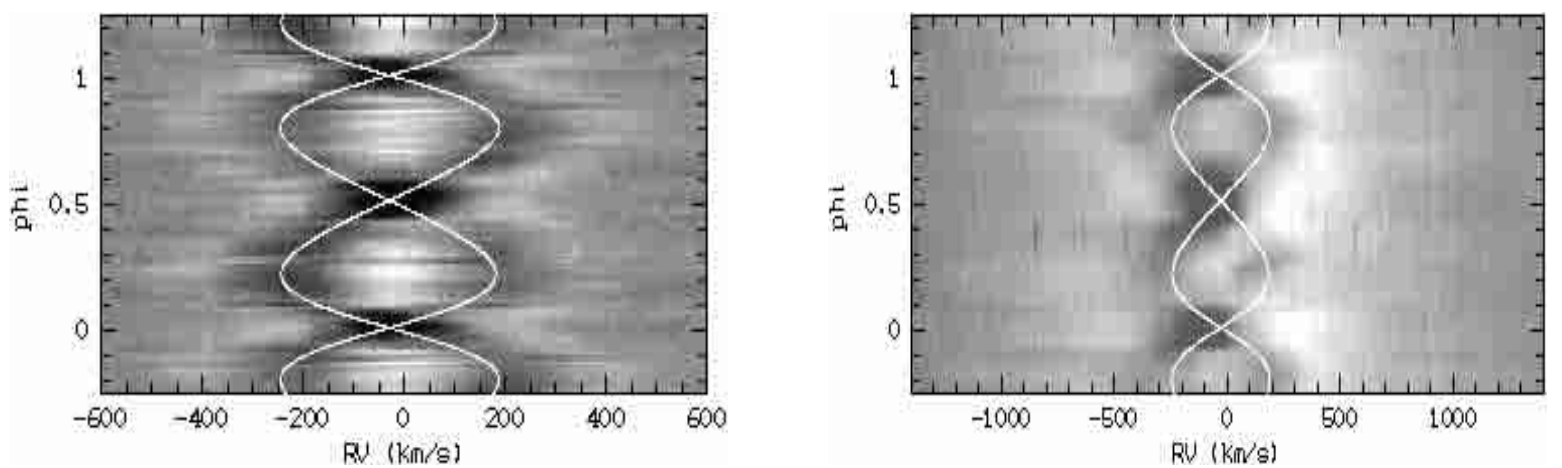

Fig. 7. Dynamical spectra of the HeII $\lambda 4686$ (left) and $\mathrm{H} \alpha$ (right) lines. The cuts are respectively set to [0.95-1.05] and [0.95-1.1]. The He I $\lambda 4471 R V$ curve has been overplotted on both panels
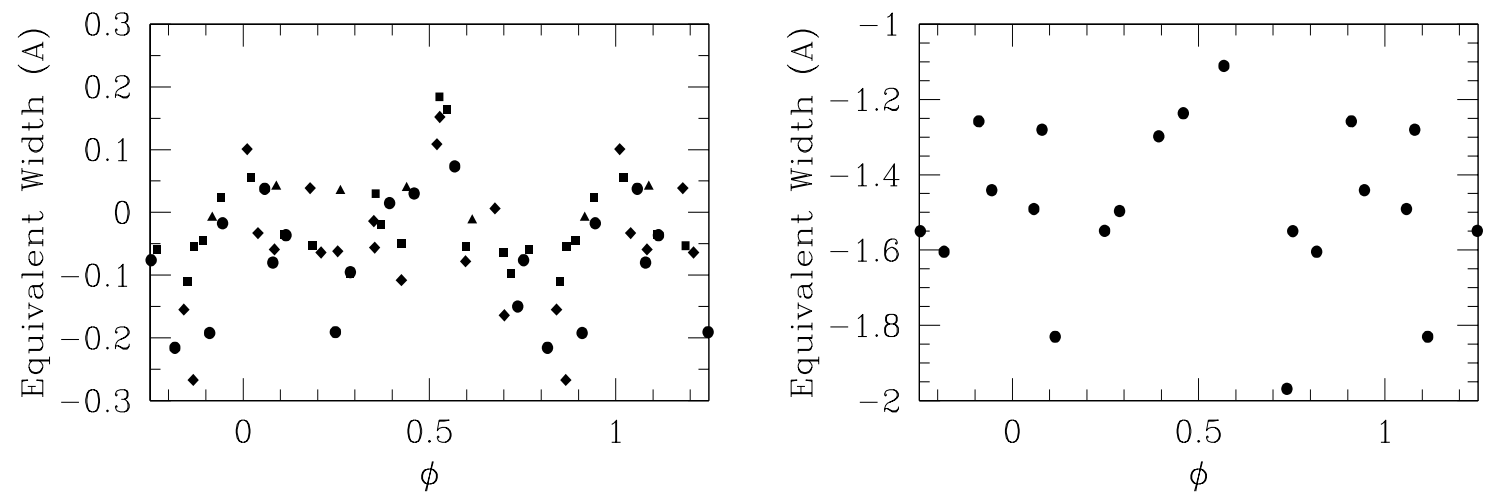

Fig. 8. Equivalent widths of the He II $\lambda 4686$ (left) and $\mathrm{H} \alpha$ (right) lines. Different symbols refer to different instruments: triangle $=\mathrm{BME}$, square $=\mathrm{B} \& \mathrm{C}$, diamond $=\mathrm{CES}$, circle $=$ FEROS
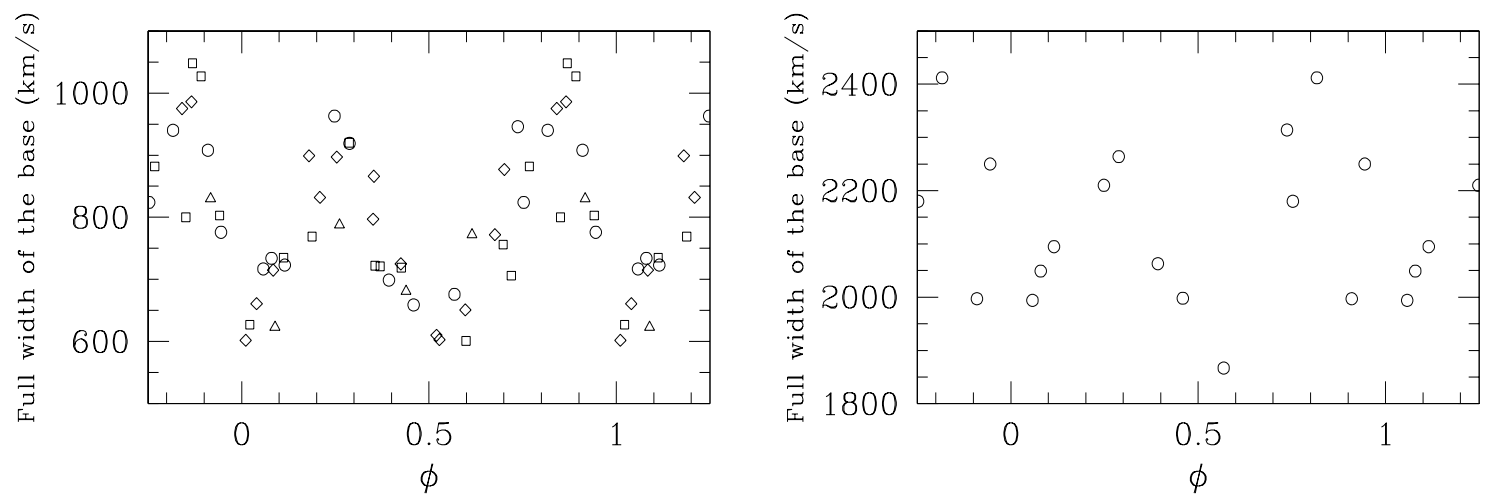

Fig. 9. Full widths of the base of the emission component of the He II $\lambda 4686$ (left) and H $\alpha$ (right) lines. Different symbols refer to different instruments: triangle $=\mathrm{BME}$, square $=\mathrm{B} \& \mathrm{C}$, diamond $=\mathrm{CES}$, circle $=\mathrm{FEROS}$

the continuum. Results are displayed in Fig. 9 and show a strong phase-locked variation of the full width at the base of the emission components. Similar measurements have been carried out on the raw observed spectra to check that our restoration method does not bias the results. Only slight differences of the order of $20 \mathrm{~km} \mathrm{~s}^{-1}$ for the start and end positions of the He II $\lambda 4686$ emission component were detected. The $\mathrm{H} \alpha$ emission component being much more intense, no significant difference could be detected for this line.

In summary, the He II $\lambda 4686$ and $\mathrm{H} \alpha$ lines behave similarly in many points. A common interpretation of the observed phase-locked modulation of both lines will be provided in Sect. 8, within the framework of a colliding wind model. However, we first need to discuss the evolutionary status of the components of HD 152248. 


\section{Evolutionary status}

\subsection{Spectral types and luminosity classes}

Following the criterion of Conti (1973), we use the logarithm of the $E W$ ratio of He I $\lambda 4471$ and He II $\lambda 4542$ to determine the spectral types of both components of the system. We get for the mean ratio:

$$
\begin{aligned}
& \log W_{\text {prim }}^{\prime}(\lambda 4471 / \lambda 4542)=0.013[-0.035,0.056] \\
& \log W_{\text {sec }}^{\prime}(\lambda 4471 / \lambda 4542)=-0.044[-0.101,0.006] .
\end{aligned}
$$

This gives a spectral type of $\mathrm{O} 7.5$ and $\mathrm{O} 7$ for the primary and secondary stars respectively. The values between brackets correspond to a $1 \sigma$ dispersion on the mean value of the $E W$ ratios. The agreement with the previous determination by $\mathrm{PGB}$ using IUE data $(\mathrm{O} 7+\mathrm{O} 7)$ is quite good.

Walborn (1972) quoted a supergiant luminosity class for the composite spectrum of HD 152248 though he noted that his classification could be vitiated because of the peculiar He II $\lambda 4686$ profile. PGB also adopted a supergiant luminosity class for both components of the system. However, the presence of He II $\lambda 4686$ and $\mathrm{H} \alpha$ in absorption in the spectrum of both stars argues against such a classification. In fact, PGB's assertion was based on the following UV criteria: a very strong emission in the Si IV $\lambda \lambda 1394-1403$ lines in all the IUE composite spectra and a blue asymmetry and red emission in the NIV $\lambda 1718$ line in both primary and secondary spectra. However, as these authors mentioned in Penny et al. (1996), the Si IV $\lambda \lambda 1394-1403$ doublet is a wind feature that might not be associated with the photosphere. Furthermore, the dependence of the N IV $\lambda 1718$ line on wind strength might lead to a more luminous classification in the case of a star with an unusually strong wind. We believe that previous confusion about the luminosity class of this system arises mainly from the fact that the lines adopted as luminosity classification criteria are probably affected by a wind interaction process occurring within the HD 152248 system (see below).

Based on the presence of He II $\lambda 4686$ and $\mathrm{H} \alpha$ in absorption in the spectrum of both components, we follow Mathys (1988) recommendation and, according to a rough determination of the $E W$ of the He II $\lambda 4686$ absorption components (from Table 7), we adopt a giant luminosity class for both stars of the HD 152248 system. Morrell et al. (1991) further showed that the unidentified $\lambda \lambda 4486$, 4504 lines display a clear luminosity dependence. An estimate of the intensity of the $\lambda 4486$ line in the spectrum of HD 152248 also suggests that the stars of HD 152248 are giants.

Finally both stars present N III $\lambda \lambda 4634-4641$ in emission so that, associated with weak He II $\lambda 4686$ absorption, an (f) tag should be added to the spectral classification. The new classification for this system is thus: O7.5III(f) + O7III(f) .

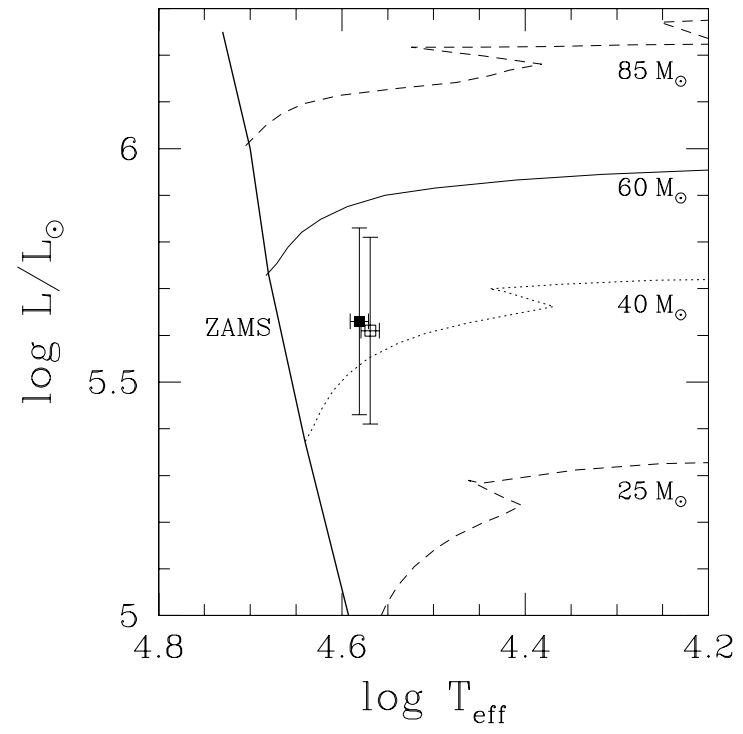

Fig. 10. Hertzsprung-Russell diagram of the HD 152248 system. The open and filled symbols stand for the primary and secondary respectively. Evolutionary tracks are from Schaller et al. (1992) for $Z=0.020$ and adopting 'standard' mass loss rates

\subsection{Location in the $H$-R diagram}

Adopting the effective temperature calibration of Chlebowski \& Garmany (1991), the spectral types derived above yield $T_{\text {eff }}=37100 \pm 1000 \mathrm{~K}$ and $T_{\text {eff }}=38100 \pm 1000 \mathrm{~K}$ for the primary and the secondary respectively. The quoted uncertainty corresponds to half a spectral subtype.

Howarth et al. (1997) and Penny et al. (1999) quote a luminosity ratio in the UV and in the visible of $I_{\text {sec }} / I_{\text {prim }} \simeq 0.98$. Raboud et al. (1997) reported $V=6.09$ and $E(B-V)=0.46$ for HD 152248 . The photometry of HD 152248 is affected by the presence of a nearby visual companion that is about $2.0 \mathrm{mag}$ fainter than the close binary (Mason et al. 1998). The values of the distance modulus of the NGC 6231 cluster range from 10.7 to 11.6. In the following, we will adopt the value $D M=11.2$ derived by Raboud et al. (1997). We thus obtain $\log \left(L_{\mathrm{bol}}^{\text {prim }} / L_{\odot}\right)=$ $5.61 \pm 0.2$ and $\log \left(L_{\mathrm{bol}}^{\mathrm{sec}} / L_{\odot}\right)=5.63 \pm 0.2$ for the primary and secondary respectively. The uncertainties on the quoted luminosities correspond to the sole uncertainty (0.5 dex) on the distance modulus of NGC 6231.

The locations of the components of HD 152248 are plotted in a H-R diagram in Fig. 10 together with the evolutionary tracks of Schaller et al. (1992). From the above results, we can infer radii of $R_{1}=15.4_{-3.1}^{+3.9} R_{\odot}$ and $R_{2}=14.9_{-3}^{+3.9} R_{\odot}$. These radii overlap within the errors with the values derived by PGB (13.4 and $12.9 R_{\odot}$ for the primary and secondary respectively) and Mayer et al. (15.6 and $17.1 R_{\odot}$ for the primary and secondary respectively). Our values of the radii are slightly larger than the typical radii of O7-O7.5 giants as listed by Howarth \& Prinja (1989), but they are definitely smaller than the radii of luminosity class I stars of same spectral type 
0.80

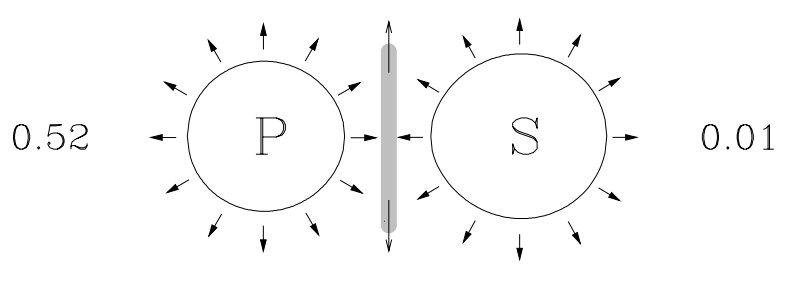

0.22

Fig. 11. Schematic view of the wind-wind interaction process occurring within the HD 152248 system. The shaded area represents the high density, emitting region. The $\mathrm{P}$ and $\mathrm{S}$ letters refer respectively to the primary and secondary components of the system. Conjunction and quadrature phases have been indicated. The adopted value for the separation between the two components is $53.1 R_{\odot}$, corresponding to $i=67.2^{\circ}$, and the shapes of the stars have been computed assuming mean radii $R_{1}=15.6 R_{\odot}$ and $R_{2}=17.1 R_{\odot}$ (Mayer et al. 2001) respectively

(Howarth \& Prinja 1989) lending further support to our assertion that the components in HD 152248 are giants rather than supergiants.

From a crude interpolation between the evolutionary tracks of Schaller et al. (1992), we derive "theoretical" masses of $M_{1}=41.7_{-6.1}^{+10.3} M_{\odot}$ and $M_{2}=43.7_{-6.1}^{+10.1} M_{\odot}$. If we adopt the inclination of $67.2^{\circ}$ as determined by Mayer et al. (2001), the minimal masses reported in Table 3 yield observed masses of 29.6 and $29.9 M_{\odot}$ for the primary and the secondary respectively. These latter values are significantly smaller than the masses predicted from the theoretical models for stars at the same location in the HR diagram. This problem was already pointed out by PGB. Improved stellar evolution models including the effects of rotation (Meynet \& Maeder 2000) might provide a solution for this mass discrepancy. In fact, the new models discussed by Meynet \& Maeder (2000) predict a large scatter in the mass-luminosity relation that can amount to a difference of $30 \%$ in mass. However, we caution that the components of HD 152248 do not show an exceptionally large projected equatorial rotational velocity (see Howarth et al. 1997).

Another way to account for this mass discrepancy consists to postulate a Roche lobe overflow scenario that could have altered the evolution of the components. Indeed the stars are close to their stability limit near periastron passage and mass loss could be initiated at that time. However, we did not find any evidence that such a process is currently taking place in HD 152248. The stars might, however, have been transferring mass through the $L_{1}$ point in the past, though the amount of transferred mass as well as the reason why this process came to a stop are unclear. Finally, we also mention Gayley's suggestion (Gayley 2001) that non-conservative mass transfer prior to the RLOF phase might exist in highly eccentric close binaries. As this is still a "working idea", we refer to his paper for more details.

\section{Wind-wind interaction}

We now attempt to provide a consistent explanation to all our observations. It is obvious, from the results presented above, that both components of the HD 152248 system are very similar. They indeed have similar polar radii, masses, luminosities and spectral types. Therefore, we can expect that the stars also have similar wind properties (mass loss rates and asymptotic velocities). Howarth et al. (1997) derived terminal velocities of $v_{\infty, 1}=2420 \mathrm{kms}^{-1}$ and $v_{\infty, 2}=2010 \mathrm{~km} \mathrm{~s}^{-1}$ for the primary and secondary respectively. We can use the stellar parameters derived above in conjunction with the theoretical mass loss recipe of Vink et al. (2000) to estimate the mass-loss rates of the two components. In this way, we obtain $\log \dot{M}_{1}=-5.53$ and $\log \dot{M}_{2}=-5.48\left(M_{\odot} \mathrm{yr}^{-1}\right)$ yielding a wind momentum ratio (Stevens et al. 1992) of

$\mathcal{R}=\left(\frac{\dot{M}_{1} v_{\infty, 1}}{\dot{M}_{2} v_{\infty, 2}}\right)^{1 / 2} \simeq 1.03$

From these simple arguments, we expect therefore that the stagnation point of a wind-wind collision should be located near the center of mass of the HD 152248 system. Adopting a "standard" $\beta$ velocity law (with $\beta=1$ ), we find that, depending on the orbital separation, the winds of both stars should reach between about 0.34 and $0.50 \times v_{\infty}$ before they collide. Using these pre-shock velocities, we obtain rather low values of the cooling parameter $\chi \leq 0.02-0.15$ (see Stevens et al. 1992 for the definition of $\chi)$. Radiative cooling could therefore play an important role in the interaction region and cool the shock-heated material to temperatures where the recombination process can efficiently contribute to the formation of the $\mathrm{H} \alpha$ and He II $\lambda 4686$ emission lines.

A very simple model that can match most of the observations consists therefore in assuming the existence of a wind-wind interaction region located mid-way between both stars and limited by two roughly planar hydrodynamic shocks. This high density region, where the He II $\lambda 4686$ and $\mathrm{H} \alpha$ emission components are assumed to be produced, would (partly) be occulted at conjunction phases $(\phi=0.01$ and 0.52$)$ which explains why the absorption is overwhelming near these phases. Similarly, this simple scenario also accounts for the phase-locked variations of the width of the emission component. Indeed, we expect from our model that the inner shock region would be almost perpendicular to the axis of the system, so that the distribution of the radial velocities of the particles escaping from the wind interaction region should be broader when our line of sight is aligned with the interaction zone (i.e. around $\phi=0.22$ and 0.80 ). On the other hand, this velocity distribution should be narrower at conjunction 
phases when our line of sight is forming an angle of about $i \approx 70^{\circ}$ with the interaction region. Near conjunction, we thus expect to observe a narrower emission line than around quadrature.

Part of the difference of the relative strengthening of the emission near quadrature phases might be explained by assuming that the emission process is depending of the separation D between the two stars following some power of $1 / \mathrm{D}$. Indeed this distance is slightly larger at $\phi=0.22$ than at $\phi=0.80$. The difference between the two absorption peaks at conjunction phases might also result from a difference in the separation between the two stars since the phases $\phi=0.0$ and $\phi=0.5$ respectively coincide with the periastron and the apastron passage. The unequal depths of the primary and secondary eclipses might also account for part of this difference, as the continuum level is lower at primary eclipse and the dilution of the emission is thus weaker. We might expect that the forthcoming X-ray observations will help to address those questions and will further allow us to refine the wind-wind interaction model of the HD 152248 system.

\section{Conclusion}

We presented the results of an optical spectroscopic campaign on the HD $152248 \mathrm{O}+\mathrm{O}$ binary system. Based on a large set of medium and high resolution spectra which provides a very good phase coverage, we derived improved orbital parameters. Combining literature data with our observations, we detected the presence of an apsidal motion with a rate of about $3.4^{\circ} \mathrm{yr}^{-1}$. As this system is going to be observed with the XMM X-ray observatory, the new contemporaneous ephemerides we derived are of major importance to correctly interpret the X-ray observation of the HD 152248 binary.

We found that the previous classification of both components as supergiants is most probably erroneous and we provide a new classification as $\mathrm{O} 7.5 \mathrm{III}(\mathrm{f})+\mathrm{O} 7 \mathrm{III}(\mathrm{f})$ for the system. This leads to a better agreement between the observed radii and masses of the components and the typical values for O7-O7.5 giants. The masses we derived are, however, still significantly lower than the ones predicted by the evolutionary tracks.

Finally, we demonstrated that the He II $\lambda 4686$ and $\mathrm{H} \alpha$ line profiles result from a blend of two absorption components, associated with the stars of the system, with a broader and more intense emission component. We further showed that their profile and intensity variations are consistent with a wind-wind collision process within the system, assuming that the He II $\lambda 4686$ and $\mathrm{H} \alpha$ emissions are produced within the interaction region through the recombination process.

The probable existence of a wind-wind interaction within the HD 152248 system makes this object an even more attractive target for XMM X-ray observations. The XMM pointings are scheduled to properly cover the orbital cycle and will further allow to investigate the different time scales of the wind interaction process. Detailed comparison of these data with recent theoretical hydrodynamical simulations (e.g. Stevens et al. 1992; Pittard 2000) will provide an important test for these models. It will further help to improve our understanding of the wind-wind collision process, and more widely of the winds of hot stars in general.

Acknowledgements. We wish to thank Dr. O. Stahl and Dr. A. Kaufer for their efficient help in adapting the FEROS reduction package to our data and the referee Dr. Stahl for his suggestions that improve our manuscript. We are grateful to $\mathrm{Y}$. Nazé and Dr. J. Manfroid for their help in the reduction of the BME data. We thank Dr. J.-M. Vreux for many helpful comments and stimulating discussions. We are also grateful to Drs. P. Harmanec and P. Eenens for discussion about HD 152248 and for communicating their results prior to publication. We are greatly indebted to the Fonds National de la Recherche Scientifique (Belgium) for multiple support. This research is also supported in part by contract P4/05 "Pôle d'Attraction Interuniversitaire" (SSTC-Belgium). Partial support through the PRODEX XMM-OM and INTEGRAL Projects is also gratefully acknowledged. The SIMBAD database was consulted for the bibliography.

\section{References}

Bagnuolo, W. G. Jr., Gies, D. R., Riddle, R., \& Penny, L. R. 1999, ApJ, 527, 353

Baume, G., Vázquez, R. A., \& Feinstein, A. 1999, A\&AS, 137, 233

Cardona-Núñez, O. 1978, Ph.D. Thesis, University of Colorado, Boulder

Chlebowski, T., \& Garmany, C. D. 1991, ApJ, 368, 241

Conti, P. S. 1973, ApJ, 179, 181

Conti, P. S., Leep, E. M., \& Lorre, J. J. 1977, ApJ, 214, 759

Corcoran, M. F. 1996, Rev. Mex. A. A. Conf., 5, 54

Eggleton, P. P. 1983, ApJ, 268, 368

Gayley, K. G. 2001, in Interacting Winds from Massive Stars, ASP Conf. Ser., ed. A. F. J. Moffat, \& N. St.-Louis, in press

Heck, A., Manfroid, J., \& Mersch, G. 1985, A\&AS, 59, 63

Hill, G., Crawford, D. L., \& Barnes, J. V. 1974, AJ, 79, 1271

Hill, G. M., Moffat, A. F. J., St.-Louis, N., \& Bartzakos, P. 2000, MNRAS, 318, 402

Howarth, I. D., \& Prinja, R. K. 1989, ApJS, 69, 527

Howarth, I. D., Siebert, K. W., Hussain, G. A. J., \& Prinja, R. K. 1997, MNRAS, 284, 265

Lafler, J., \& Kinman, T. D. 1965, ApJS, 11, 216

Levato, H., \& Morrell, N. 1983, ApL, 23, 183

Luna, H. G. 1988, A\&AS, 74, 427

Mason, B. D., Gies, D. R., Hartkopf, W. I., et al. 1998, AJ, 115,821

Mathys, G. 1988, A\&AS, 76, 427

Mayer, P., Lorenz, R., \& Drechsel, H. 1992, IBVS, 3765, 1

Mayer, P., Harmanec, P., Lorenz, R., et al. 2001, in The Influence of Binaries on Stellar Population Studies, ed. D. Vanbeveren (Kluwer Academic Publisher), in press

Meynet, G., \& Maeder, A. 2000, A\&A, 361, 101

Morrell, N. I., Walborn, N. R., \& Fitzpatrick, E. L. 1991, PASP, 103,341

Nussbaumer, H. 1971, ApJ, 170, 93

Penny, L. R., Gies, D. R., \& Bagnuolo, W. G. Jr. 1996, ApJ, 460, 906 
Penny, L. R., Gies, D. R., \& Bagnuolo, W. G. Jr. 1999, ApJ, 518,450

Pittard, J. M. 2000, Ph.D. Thesis, University of Birmingham (UK)

Pittard, J. M., \& Stevens, I. R. 1997, MNRAS, 292, 298

Raboud, D., Cramer, N., \& Bernasconi, P. A. 1997, A\&A, 325, 167

Rauw, G., Vreux, J.-M., \& Bohannan, B. 1999, ApJ, 517, 416

Rauw, G., Sana, H., Gosset, E., et al. 2000, A\&A, 360, 1003

Rauw, G., Sana, H., Vreux, J.-M., Gosset, E., \& Stevens, I. R. 2001, in Interacting Winds from Massive Stars, ASP Conf. Ser., ed. A. F. J. Moffat, \& N. St.-Louis, in press

Sana, H., Rauw, G., Gosset, E., \& Vreux, J.-M. 2001, in Interacting Winds from Massive Stars, ASP Conf. Ser., ed. A. F. J. Moffat, \& N. St.-Louis, in press

Schaller, G., Schaerer, D., Meynet, G., \& Maeder, A. 1992,
A\&AS, 96, 269

Stevens, I. R., Blondin, J. M., \& Pollock, A. M. T. 1992, ApJ, 386,265

Stickland, D. J., Lloyd, C., Penny, L. R., Gies, D. R., \& Bagnuolo, W. G. Jr. 1996, Observatory, 116, 226

Struve, O. 1937, ApJ, 85, 41

Struve, O. 1944, ApJ, 100, 189

Thaller, M. L. 1997, ApJ, 487, 380

Underhill, A. B. 1994, ApJ, 420, 869

Vink, J. S., de Koter, A., \& Lamers, H. J. G. L. M. 2000, A\&A, 362,295

Walborn, N. R. 1972, AJ, 77, 312

Walder, R. 1998, Ap\&SS, 260, 243

Wolfe, R. H. Jr., Horak, H. G., \& Storer, N. W. 1967, in Modern Astrophysics, ed. M. Hack (Gordon \& Breach, New York), 251 